\title{
Failure of facet replacement system with metal-on-metal bearing surface and subsequent discovery of cobalt allergy: report of 2 cases
}

\author{
Matthew L. Goodwin, MD, PhD, William Ryan Spiker, MD, Darrel S. Brodke, MD, and \\ Brandon D. Lawrence, MD \\ Department of Orthopaedics, University of Utah, Salt Lake City, Utah
}

The aim of this study was to report on 2 patients in whom metal-on-metal (MOM) facet replacements failed, with subsequent positive findings on allergy testing. Motion-preserving devices have been used with limited success when instrumentation is indicated in the mobile spine. MOM-bearing surfaces in orthopedics were developed to increase implant longevity, yet have been associated with numerous adverse outcomes, including local tissue reactions, pseudotumors, metallosis, and the need for revision surgery. Five patients with spinal stenosis and low-grade spondylolisthesis were randomized to undergo facet replacement surgery with the ACADIA facet replacement system at the authors' institution. Two patients experienced a return of neurological symptoms after a pain-free interval (<2 years) with development of local tissue reaction and positive findings on allergy testing to cobalt, the metal in the MOM-bearing surface. Both patients underwent successful removal of the implant and revision to titanium posterior spinal fusion and interbody fusion without further complication.

Motion-preserving devices have been designed and trialed for specific indications in the mobile spine. Given the adverse results from MOM devices in hip arthroplasty and now the early reports with MOM facet replacements, caution is warranted when moving forward with any MOM joint-bearing surface. Both patients presented here had an unusual tissue reaction locally and subsequent positive allergy testing results to cobalt. These 2 patients appear to have developed a delayed hypersensitivity reaction to the metal, likely from fine debris at the MOM interface.

https://thejns.org/doi/abs/10.3171/2017.10.SPINE17862

KEYWORDS ACADIA facet replacement system; AFRS; cobalt; metal-on-metal; spondylolisthesis; metal allergy

$\mathrm{R}$ ECENTLY, interest has grown in facet replacement devices for conditions such as spinal stenosis with instability. ${ }^{8}$ Our institution participated in a randomized controlled trial studying the ACADIA facet replacement system (AFRS; Globus Medical, http://www. globusmedical.com/acadia-ide-study/), a pedicle-based device with a metal-on-metal (MOM) gliding joint that mimics the native facet biomechanics (clinical trial registration no. NCT00401518, clinicaltrials.gov) (Fig. 1). Five patients with spinal stenosis and low-grade spondylolisthesis were randomized to undergo facet replacement surgery with the AFRS at our institution. Here, we present the cases of 2 patients who developed delayed symptoms and local tissue reactions, which have not been previously described. Both patients subsequently tested positive for metal allergies to cobalt and underwent removal of the implant with conversion to posterior spinal instrumented fusion (PSIF) with interbody fusion.

\section{Case Reports}

Case 1

A 59-year-old man presented with several years of worsening back and bilateral radicular leg pain and neurogenic 


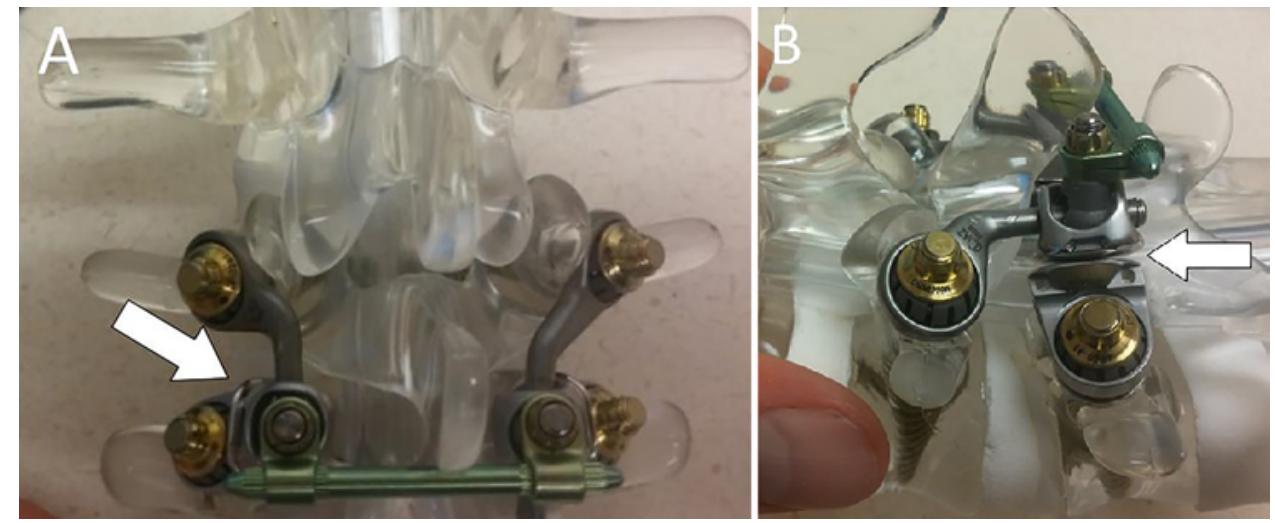

FIG. 1. Posterior (A) and sagittal (B) views of a model of the AFRS. Note the MOM-bearing surface (arrow). Figure is available in color online only.

claudication for which conservative treatment measures had failed. The patient had grade 1 spondylolisthesis at L4-5 with MRI evidence of central and foraminal stenosis. After consent and randomization, he underwent a wide laminectomy with facet resection and AFRS, which was followed by a complicated postoperative course, including prostatitis, drug reaction with eosinophilia and systemic symptoms, and diverticulitis requiring colon resection. Eight months postoperatively, the patient developed recurrent pain and claudication, weakness in the left extensor hallucis longus muscle (4/5), and recurrent stenosis. Revision surgery to an L4-5 PSIF with interbody fusion and removal of the AFRS revealed a chalky, soft scar without frank metallosis (Fig. 2A-C). The patient was found to have a severe cobalt allergy on subsequent allergy testing.

\section{Case 2}

A 69-year-old woman presented with a 3-month history of radicular right leg pain and neurogenic claudication that was refractory to conservative measures. The patient's examination findings were remarkable for weak (4/5) ankle dorsiflexion bilaterally. She had grade 1 degenerative spondylolisthesis at L4-5 with MRI evidence of central and foraminal stenosis. After consent and enrollment, the patient was randomized to and underwent laminectomy, facet resection, and AFRS without complication. At her 3and 6-month postoperative visits, the patient had complete pain relief with resolution of her extensor hallucis longus muscle weakness. Around 1.5 years postoperatively, the patient had increasing pain with weakness (4/5) and atrophy in her quadriceps muscles. Her spondylolisthesis had increased to grade 2, and MRI showed a large, soft-tissue/ fluid collection, which was concerning for local tissue reaction with recurrent stenosis. Allergy testing revealed a delayed allergic reaction to cobalt. The patient underwent uneventful revision to an L4-5 PSIF with interbody fusion and removal of the AFRS. Intraoperatively, serous fluid and a chalky, soft scar without frank metallosis were noted (Fig. 2D-F).

\section{Discussion}

Particularly for single-level disease, facet replacement is an appealing option for patients who have lumbar steno- sis and facet hypertrophy and nonoperative management has failed. Facet replacement in this scenario allows for stable decompression and facetectomy without the need for fusion (i.e., loss of motion). Here, we have presented the cases of 2 patients in whom facet replacement had failed; had the same unique chalky, yogurt-like, soft scar that we have not previously seen; and were subsequently found to have cobalt allergies. Both patients had this unique scar formation rather than the typical ligamentous/capsular hypertrophy seen in other revision cases.

Allergy to metal has been previously described in the spine literature, ${ }^{9}$ but not in regard to MOM-bearing surfaces. One case report described a reaction to a titanium implant, including a similar clinical course with a period of relief followed by return of symptoms months later and subsequent allergy on testing. ${ }^{9}$ Given the overall low incidence of allergy to cobalt in the general population (approximately 1\%-2\%), ${ }^{7}$ it is surprising that 2 of our 5 patients would demonstrate allergies to cobalt on patch testing. In the $1970 \mathrm{~s}$, Nater et al. ${ }^{5}$ patch tested patients before and after implantation of a metal-on-plastic total hip arthroplasty. No hips failed, yet several patients developed hypersensitivity, suggesting that wear from even a well-tolerated total hip arthroplasty is enough to induce a positive patch test. ${ }^{5}$ Given the MOM-bearing surface and the classic pain-free interval after surgery but before symptoms appear, ${ }^{10}$ it is probable that fine debris particles led to a type IV delayed hypersensitivity reaction in our 2 patients. Furthermore, the results now available after hip arthroplasty suggest that many asymptomatic patients with MOM implants can be closely monitored rather than undergo revision prophylactically. ${ }^{6}$ The remaining patients with MOM facet replacements in our cohort are being closely followed clinically. Given the data from the study by Nater et al., ${ }^{5}$ positive results on an allergy test in a patient who was clinically asymptomatic would not warrant revision surgery.

Reactions occurring with MOM implants have been described in various ways, including inflammatory pseudotumor (aseptic cyst in reaction to debris), aseptic lymphocytic vasculitis-associated lesion, metallosis (aseptic fibrosis, local necrosis), ${ }^{2}$ and metal toxicity. ${ }^{1}$ Both of our patients presented in a delayed fashion with a soft, chalky, yogurt-like scar with some cystic component (Fig. 3) but 

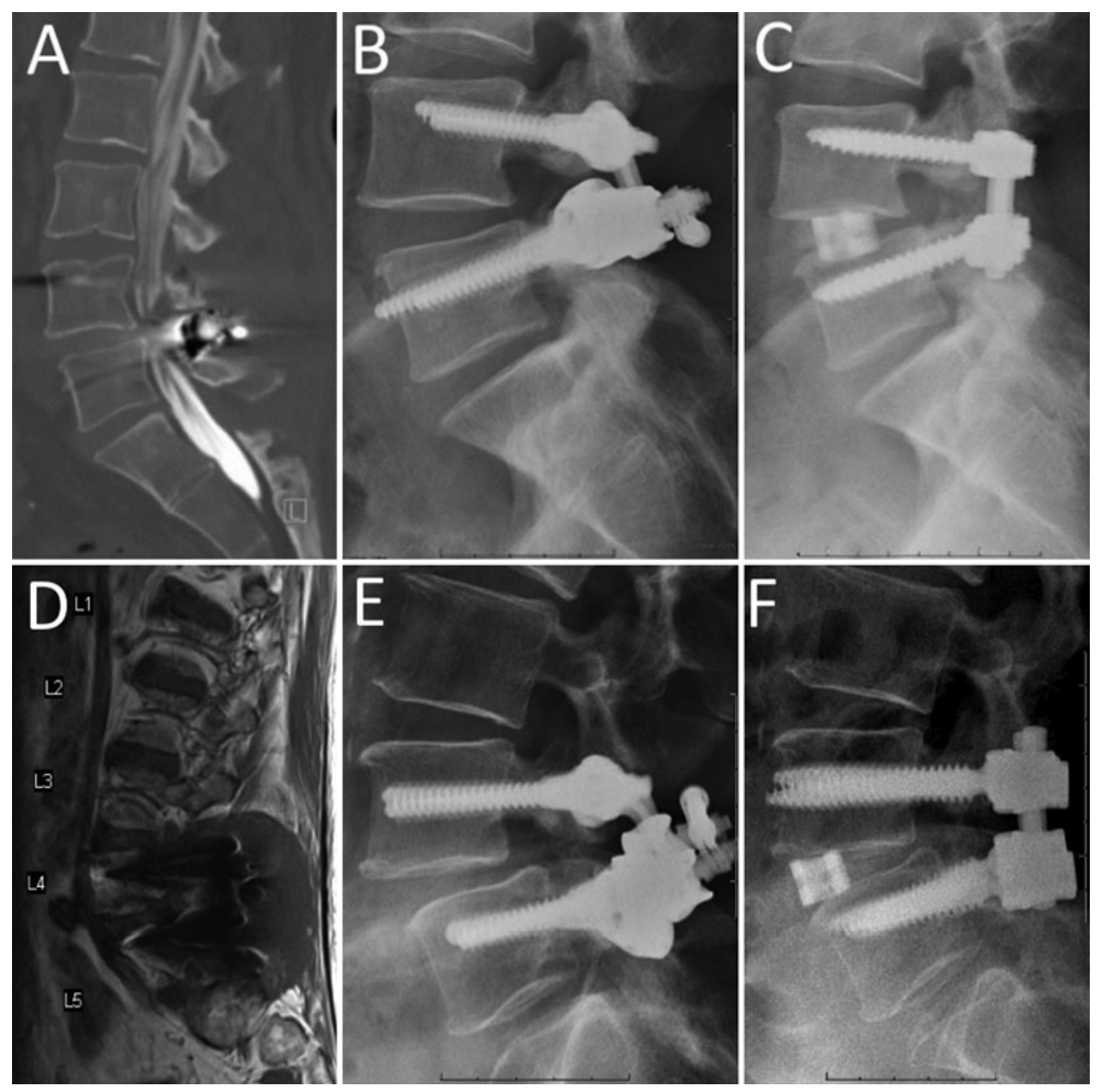

FIG. 2. A-C: Case 1. CT myelogram showing restenosis (A). Preoperative (B) and postoperative (C) radiographs obtained after the revision surgery to PSIF with interbody fusion and removal of the facet replacement. D-F: Case 2. MR image showing the reactive mass $(D)$. Preoperative $(E)$ and postoperative $(F)$ images obtained after the revision surgery to PSIF with interbody fusion and removal of the facet replacement.

without frank metallosis. At a recent conference, a colleague presented the case of a patient with a failed total disc replacement and a similar clinical course and similar unique intraoperative scar consistency, but allergy testing was not performed. The scar described by this colleague

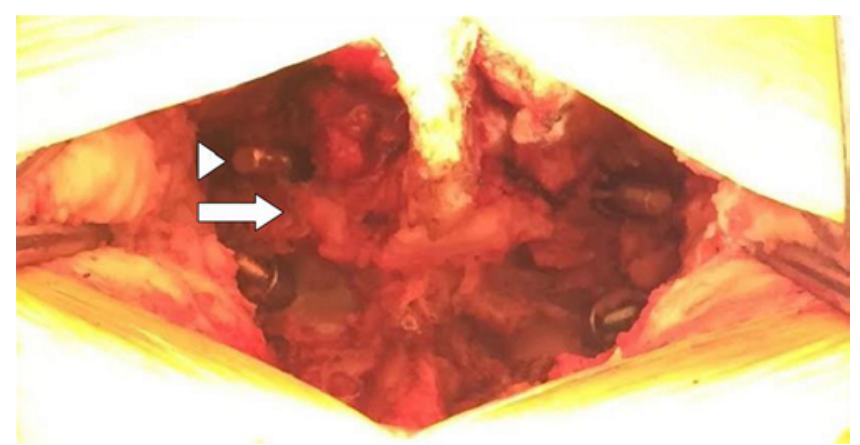

FIG. 3. Characteristic soft, yogurt-like, chalky, white scar seen in both cases (arrow). The arrowhead points to a pedicle post after removal of the AFRS. Figure is available in color online only. and in previous reports of failed total disc replacements ${ }^{3}$ is not unlike what we encountered. While our allergy testing was done via patch testing, some have argued that the leukocyte transformation test is more "physiological;", neither test is likely to completely represent the host-metal implant environment (Fig. 3).

In conclusion, facet replacements with MOM-bearing surfaces failed in 2 of our 5 patients; both patients had a delayed hypersensitivity reaction to the cobalt, likely due to metal debris. In both cases, the patients underwent uneventful revisions to traditional titanium constructs with interbody cages without further complication (Fig. 2C and F).

\section{Acknowledgments}

We thank Dr. Jeremy M. Gililland for helpful comments and discussions on MOM complications.

The device that is the subject of this article is being evaluated as part of an ongoing FDA-approved investigational protocol (investigational device exemption) or corresponding national protocol to evaluate the safety and effectiveness of the AFRS compared with an instrumented posterolateral fusion control. 


\section{References}

1. Allen LA, Ambardekar AV, Devaraj KM, Maleszewski JJ, Wolfel EE: Clinical problem-solving. Missing elements of the history. N Engl J Med 370:559-566, 2014

2. Drummond J, Tran P, Fary C: Metal-on-metal hip arthroplasty: a review of adverse reactions and patient management. $\mathbf{J}$ Funct Biomater 6:486-499, 2015

3. Guyer RD, Shellock J, MacLennan B, Hanscom D, Knight $\mathrm{RQ}$, McCombe P, et al: Early failure of metal-on-metal artificial disc prostheses associated with lymphocytic reaction: diagnosis and treatment experience in four cases. Spine (Phila Pa 1976) 36:E492-E497, 2011

4. Hallab NJ, Jacobs JJ: Biologic effects of implant debris. Bull NYU Hosp Jt Dis 67:182-188, 2009

5. Nater JP, Brain RG, Deutman R, Mulder TJ: The development of metal hypersensitivity in patients with metal-toplastic hip arthroplasties. Contact Dermat 2:259-261, 1976

6. Roberts TT, Haines CM, Uhl RL: Allergic or hypersensitivity reactions to orthopaedic implants. J Am Acad Orthop Surg 25:693-702, 2017

7. Schäfer T, Böhler E, Ruhdorfer S, Weigl L, Wessner D, Filipiak B, et al: Epidemiology of contact allergy in adults. Allergy 56:1192-1196, 2001

8. Serhan H, Mhatre D, Defossez H, Bono CM: Motion-preserving technologies for degenerative lumbar spine: The past, present, and future horizons. SAS J 5:75-89, 2011

9. Shang X, Wang L, Kou D, Jia X, Yang X, Zhang M, et al: Metal hypersensitivity in patient with posterior lumbar spine fusion: a case report and its literature review. BMC Musculoskelet Disord 15:314, 2014

10. Thomas P: Clinical and diagnostic challenges of metal implant allergy using the example of orthopaedic surgical implants: Part 15 of the Series Molecular Allergology. Allergo J Int 23:179-185, 2014

\section{Disclosures}

Globus Medical Inc. provided funding for this study. Dr. Spiker: consultant for NEXXT Orthopaedics and K2M, and fellowship support from DePuy Synthes.

\section{Author Contributions}

Conception and design: all authors. Acquisition of data: Lawrence, Spiker, Brodke. Analysis and interpretation of data: Lawrence, Spiker, Brodke. Drafting the article: all authors. Critically revising the article: Lawrence, Spiker, Brodke. Reviewed submitted version of manuscript: all authors. Approved the final version of the manuscript on behalf of all authors: Lawrence. Administrative/technical/material support: all authors. Study supervision: all authors.

\section{Correspondence}

Brandon D. Lawrence: University of Utah, Salt Lake City, UT. brandon.lawrence@hsc.utah.edu. 